\title{
Gut geleitet, profund positioniert
}

L

eitlinien, Positionspapiere, Positionspapiere, Leitlinien - das Bemühen um gesichertes Wissen und seine Anwendung bei der Versorgung des Patienten war noch nie so groß! Das unbestritten wichtige Ziel dieser Anstrengungen sind die Schaffung und Sicherung bestmöglicher Qualität ärztlichen Handelns.

Fraglich ist allerdings, ob die qualitätssichernden Botschaften die Handelnden wie beabsichtigt erreichen. Es gibt Handbücher, es gibt Fachzeitschriften, es gibt Fortbildungsveranstaltungen steht das in Positionspapieren oder Leitlinien dargelegte Wissen, das ja auch aus diesen Quellen stammt, darüber? Oder wollen sich hier etwa beckmesserische Wichtigtuer profilieren? Und: Steht der Nutzen qualitätssichernder Papiere in einem sinnvollen Verhältnis zum enormen Aufwand an Zeit und Geld, den ihre Erstellung erfordert - eine Leitlinie der Entwicklungsstufe drei kostet ca. 150.000 Euro!

Wir alle wissen: Der

„Positionspapiere und
Leitlinien sind die Seile,
die uns im Treibsand
des verfallen(d)en
Wissens und des
aktuellen Wissens
sichern können.“

entwicklung selbst kontinuierlich zu verfolgen. Auch können Leitlinien individuelle ärztliche Entscheidungen bei der Patientenversorgung nicht ersetzen - sie sind „rechtlich nicht bindend und haben daher weder haftungsbegründende noch haftungsbefreiende Wirkung" (Arbeitsgemeinschaft der Wissenschaftlichen Medizinischen Fachgesellschaften). Vor allem auch sind Leitlinien keine Anleitung zur möglichst billigen Reparatur der Biomaschine Mensch, ein ökonomischer Nutzen Zuwachs an medizinischem Wissen und ärztlichen Handlungsmöglichkeiten in Diagnostik und Therapie ist immer rascher geworden. Parallel dazu verlieren hergebrachte Vorstellungen und Vorgehensweisen ihre Gültigkeit. Selbst der gut informierte Spezialist ist kaum noch in der Lage, die Vielfalt an Informationen auf seinem Gebiet aufzunehmen oder gar in ihrer Gesamtheit zu werten.

Positionspapiere und Leitlinien, die von ärztlichen Expertengruppen erstellt und fortlaufend aktualisiert werden, helfen wesentlich dabei, auf der Höhe des Wissens zu bleiben. Die Berücksichtigung qualitätssichernder Papiere entbindet jedoch nicht davon, die Wissens- kann sich jedoch aus der Vermeidung falschen Handelns ergeben.

Positionspapiere und Leitlinien sind die Seile, die uns im Treibsand des verfallen(d)en Wissens und des aktuellen Wissens am Grunde des Meeres des Nicht-Wissens sichern können. Es ist klar: Die offensichtlich unbegrenzte Zunahme wissenschaftlicher Erkenntnisse und daraus abgeleiteter Diagnos-

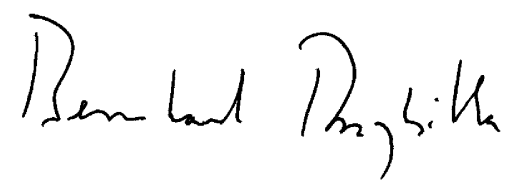

Prof. Dr. B. Przybilla tik- und Therapieverfahren bedeutet, dass auch unser Nicht-Wissen - optimistisch Noch-Nicht-Wissen - unbegrenzt ist. Der Kranke kann nicht warten, bis dies anders ist - aber er hat Anspruch auf das aktuell bestmögliche Wissen!

Ein Positionspapier der DGAI zu einem wichtigen Bereich der Allergologie finden Sie auch in diesem Heft des Allergo Journal (Seite 492): Die Ausführungen zur In-vitro-Allergiediagnostik sind umfassend, die Umsetzung in die klinische Praxis erfordert die erwähnte individuell wertende Vorgehensweise. Besondere Vorsicht ist vor allem dort nötig, wo Hypothesen ins Kraut schießen, durch ihre bloße Existenz zu „Wissen“ mutieren und dann zu unbegründeten, gar gefährdenden therapeutischen Konzepten führen. Die kritische Auseinandersetzung mit der heute so beliebten Hygiene-Hypothese als Erklärung für die Zunahme allergischer Erkrankungen macht deutlich, dass praktische Handlungsanleitungen aus dieser - wissenschaftlich unbestritten attraktiven Hypothese! - bisher nicht abzuleiten sind (Seite 507). Die Anwendung rekombinanter Allergene ist eine zukunftweisende Entwicklung in der Allergologie: Schon jetzt können mit diesen Allergenen Testverfahren verbessert werden, ihr späterer Einsatz auch in der Therapie ist viel versprechend (Seite 513).

Wir wünschen Ihnen ruhige und besinnliche Festtage und ein glückliches neues Jahr!

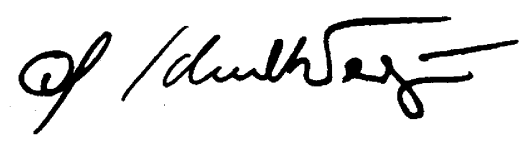

Prof. Dr. G. Schultze-Werninghaus 